\title{
Treatment of spontaneous intracranial hypotension: evolution of the therapeutic and diagnostic modalities
}

\author{
Angelo Franzini - G. Messina - L. Chiapparini • \\ G. Bussone
}

(C) Springer-Verlag Italia 2013

\begin{abstract}
Magnetic resonance imaging in patients affected by orthostatic headache often allows the diagnosis of spontaneous intracranial hypotension (SIH). Nevertheless, in the last 5 years, the diagnostic and therapeutic strategy for spontaneous intracranial hypotension $(\mathrm{SIH})$ has been significantly modified. Specifically, all invasive techniques aimed at localization and demonstration of "spontaneous" spinal fistulas (myelography, isotopic cisternography, and so on) have been progressively abandoned. Also myelo$\mathrm{MR}$, although not an invasive exam, is no longer considered necessary to establish the most appropriate treatment. This change is due to the development and demonstration of a pathogenetic theory which considers the cerebrospinal fistula not as the primary cause of intracranial hypotension, but as the consequence of an imbalance between epidural and cerebrospinal fluid (CSF) pressures. In a consecutive series of 80 patients, we performed a standardized epidural lumbar injection of a dense compound aimed to raise the epidural pressure irrespective of the presence and site of CSF leaks. The technique used, the long-term results of this treatment and the diagnostic pathways will be discussed.
\end{abstract}

\footnotetext{
A. Franzini $(\bowtie) \cdot$ G. Messina

Department of Neurosurgery, Neurological Institute "C. Besta",

Via Celoria, 11, 20133 Milan, Italy

e-mail: angelo@angelofranzini.com

L. Chiapparini

Department of Neuroradiology, Neurological Institute

"C. Besta”, Milan, Italy

G. Bussone

Department of Neurological Sciences, Headache Unit Fondazione IRCCS, Istituto Neurologico "C. Besta", Milan, Italy
}

Keywords Spontaneous intracranial hypotension - SIH * Blood patch · Orthostatic headache

\section{Introduction}

Hospital admissions for spontaneous intracranial hypotension (SIH) resistant to conservative treatments have increased in the last 5 years, growing from sporadic cases to more than 20 cases per year in our institute. Such increase is due to a better awareness of this syndrome $[9,10]$ and to an easier access to magnetic resonance imaging (MRI) examinations. SIH typically presents with postural orthostatic headache [8] which in the most severe conditions may be associated with nausea, vomiting, dizziness, diplopia, photophobia, hearing impairment, neck stiffness, blurred vision, sometimes behavioural disorders or/and abnormal movements.

MRI of the brain with gadolinium injection is the imaging study of choice for patients with clinical suspect of SIH. Characteristic radiologic abnormalities [2-7] include diffuse thickening and contrast enhancement of the pachymeninges, subdural fluid collections or subdural hematomas that are frequently bilateral (Fig. 1).

In the last 5 years, in our institute important changes of diagnostic pathways have been introduced. Specifically, all invasive techniques aimed at localizing and demonstrating "spontaneous" spinal fistulas (myelography, isotopic cisternography, and so on) have been progressively abandoned.

Also myelo-MR, although not an invasive examination, is not routinely performed. This strategy is due to the development of a novel aetiopathogenetic theory which considers the cerebrospinal fistula not as the primary cause of the intracranial hypotension, but as the consequence of an imbalance between epidural and cerebrospinal fluid (CSF) pressures [5]. 

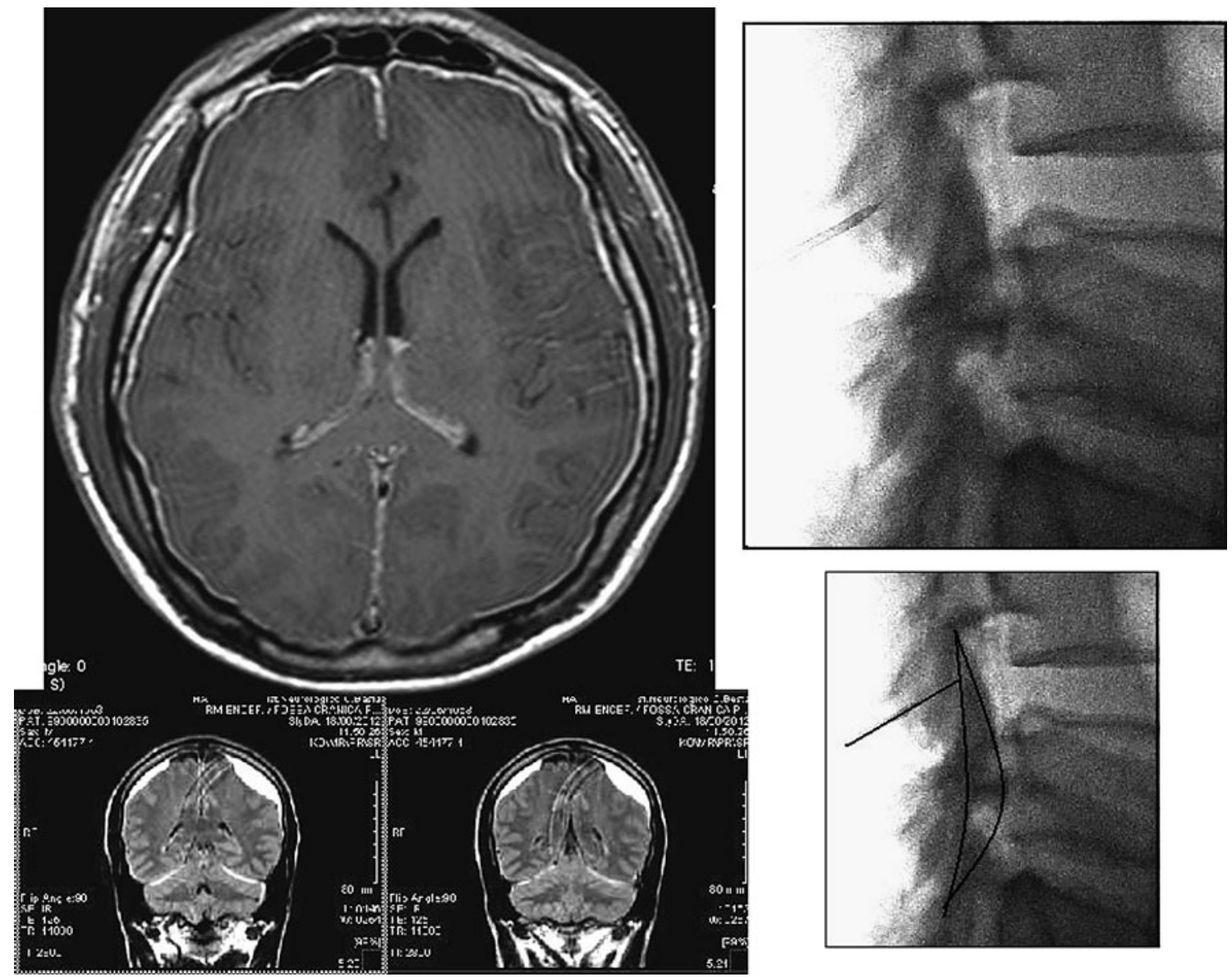

Fig. 1 Left Axial (upper) and two coronal (lower) MRI images of two patients with SIH; notice the pachymeningeal contrast enhancement in the axial image and the subdural fluid collections in the coronal images. Right Lateral intra-procedural radiographic image showing the Tuoy needle approximating the interlaminar space of two consecutive lumbar vertebrae (upper), and the schematic localization of the blood patch compound (lower) once injected into the epidural space
In 80 consecutive patients we demonstrated that, by provoking an increase in epidural pressure by injecting a dense compound into the lumbar epidural space, it is possible to obtain the recovery from this syndrome, irrespective of the demonstration and/or localization of CSF fistulas.

The technique, the results of treatment and the diagnostic-therapeutic strategy will be in relation to the hypothesis of the epidural/intradural pressures imbalance.

\section{Physiopathological hypothesis}

Our hypothesis is based on the anatomy and physiology of the epidural veins as well as on the observation of an abnormally low spinal epidural pressure observed in patients affected by SIH.

Venous drainage of the spinal epidural space is served by two main anatomical complexes. The upper thoracic plexiform venous network drains into the superior vena cava system via the radicular veins. Below L-2, the lumbar epidural venous network drains into the inferior vena cava system via a network of large radicular veins. These two systems communicate at the thoracolumbar junction [5]. Anti-reflux venous valves protect the spinal cord from the high-pressure venous waves of the vena cava system, but cannot prevent abnormally augmented venous drainage from the lumbar epidural veins. Increased venous drainage from the lumbar epidural venous system to the inferior vena cava will result in reduced volume of the emptied epidural veins and this will cause increase of the negative pressure within the epidural space, which constitutes a closed system. The negative pressure of the epidural compartment acts as an aspiration force applied to the whole dural surface, and in our opinion, will be responsible of CSF steal through the dura itself or through provoked fistulas where the 
dura is particularly thin or fenestrated (such as in the cervical radicular pockets).

Low venous pressure within the inferior vena cava system is amplified by the muscles pumping blood during standing and walking and these actions are well known to worsen SIH.

Following these considerations, we argued that a rise in the epidural pressure would abolish the "aspiration" effect on the dural surface and so, also abolish the CSF steal. In other words, only a significant pressure gradient between the intradural pressure (positive) and the epidural pressure (negative) will maintain the CSF outflow and CSF loss trough the venous system. When the gradient between the two compartments is nill, the passage of CSF will also be nill. So we decided to introduce within the epidural lumbar space a dense compound aimed to obtain the increase in the epidural pressure and a partial segmental compression of the lumbar epidural vein to obtain further and stable reduction of the venous drainage to the inferior cava system. Our hypotheses have been extensively published and discussed [5] and is schematically represented in Fig. 2. The following considerations are stressed:

- The site of the CSF leak is not influential because in any case the cause of the disease is the negative epidural pressure maintained by the lumbar venous drainage toward the inferior cava system.

- The conventional blood patch or the blood patch in the Trendelenburg position is effective [1, 3, 4, 11], because the injection of blood within the epidural space modifies the pressure gradients between the epidural and intradural compartments but, in our opinion, it is not necessary that blood actually reaches the site of the fistula [6].

- The optimal procedure would be aimed at increasing the epidural pressure instead of closing the CSF leak.

So we introduced a blood patch procedure based on the injection of a dense compound within the lumbar epidural space, irrespective of the actual site of the CSF leak [5]. Following the first results in a preliminary series of 28 patients and the demonstration that the lumbar procedure was effective in presence of cervical demonstrated CSF leaks [6], we definitively abandoned all the diagnostic procedures aimed at searching the site of CSF leaks and introduced a standard procedure for all patients affected by SIH.

\section{Patients and methods}

Technique

Five $\mathrm{ml}$ of fibrin glue is mixed with $5 \mathrm{ml}$ of homologous blood and $3 \mathrm{ml}$ of hydrosoluble contrast medium. The resulting viscous compound is then injected into the epidural space at L1-2 or lower levels under fluoroscopic control with the patient lying prone. The epidural needle connected to a syringe filled with saline solution is left in place for a few minutes to balance the epidural pressure with the atmospheric pressure $(\sim 15-20 \mathrm{ml}$ of saline is usually aspirated spontaneously within the epidural space due to the negative epidural pressure). Then the compound is injected under fluoroscopic guidance (Fig. 1).

The day after the procedure the patient can be discharged from hospital and will return to normal working and social activities in few weeks.

\section{Patient series}

Between 2000 and 2011, 80 patients (mean age 42, range 22-60 years, 44 females) have been admitted to our institution because of SIH resistant to conservative treatments for at least 2 months.

In all patients the heralding and prominent symptom of the disease was headache and/or neck pain appearing or worsening within $15 \mathrm{~min}$ after sitting or standing. In addition to these symptoms, some patients complained nausea (19 patients), tinnitus (29 patients), hypoacusia (45 patients). Mild endocrine dysfunction related to $\mathrm{ADH}$ deficiency was present in two cases. One patient complained a severe behaviour disorder with anhedonia, apathia and depression while one patient complained parkinsonian rest tremor (both patients recovered from symptoms few weeks after the blood patch procedure). The median time elapsed between symptom's onset and diagnosis was 4 months (from 15 days to 45 months).

Brain MRI features were: diffuse thickening and linear enhancement of the pachymeninges along the convexities, the cerebral falx, the tentorium and the clivus (79 cases), intradural fluid collections or hematomas (15 cases), sagging of the brain with caudal diencephalic displacement, descent and enlargement of the pons, flattening of the peripontine cisterns and, in some case, downward tonsillar displacement (6 cases). Additional findings included pituitary enlargement and engorgement of the dural venous sinuses. One of these findings was observed in almost all the patients of this series.

In most of the patients spinal MRI findings included: epidural fluid collections within the spinal canal and engorgement of the venous plexuses in the cervical epidural anterior space. The search for CSF leak has been performed by MRI myelograms in 39 patients and by isotopic myelography in 14 cases. The CSF leak has been demonstrated in four patients within the cervical (2 patients), thoracic (1 patients) and lumbar (1 patient) spinal segments. In these series the search for the CSF 

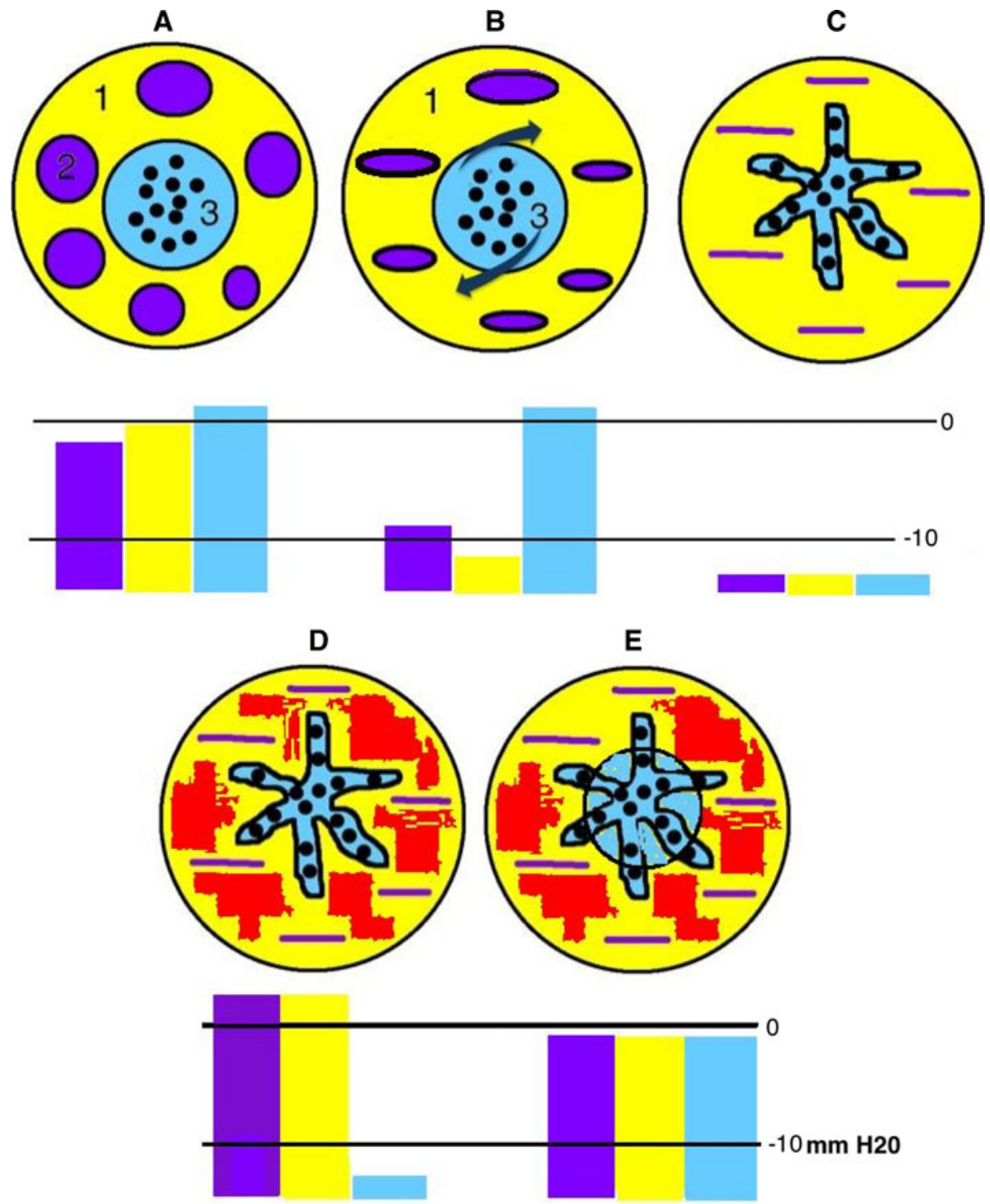

Fig. 2 Schematic representation of the three spinal compartments as axial spinal lumbar sections: violet veins, yellow epidural space, blue CSF, black dots roots, red blood patch compound At the bottom are represented the relative pressures of the considered compartments with the same colour code. a Steady state of the normal condition with volumes (upper) and pressures (bottom) in equilibrium. b In conditions of abnormal venous drainage the veins are emptied by the blood aspiration from the cava system and veins volume is reduced (upper) provoking a negative pressure in the spinal closed system. CSF leaves the dural sac due to the existing pressure gradient between the intradural positive pressure compartment and the epidural negative pressure compartment (represented at the bottom of the

leaks was mainly performed between 2000 and 2005, while in the last 5 years diagnostic workup has gradually been modified and spinal studies have been abandoned because of the adoption of the above-mentioned standardized blood patch procedure always being performed within the lumbar region. figure). $\mathbf{c}$ The CSF that escaped from the dural sac is absorbed by the epidural veins and leaves definitively the system through the inferior vena cava system. The steal of CSF is responsible of the volume reduction of the intradural compartment and is so responsible for the whole syndrome. $\mathbf{d}$ The dense compound injected within the epidural space (the so called "blood patch") rises the epidural pressure (yellow column at bottom of the figure) inverting the pressure gradient between the intradural and epidural compartments $\mathbf{e}$ The veins (violet) remain collapsed by the blood patch and the new CSF production gradually replaces the CSF steal. In this phase hydration and steroids may be helpful to increase and restore CSF volume (colour figure online)

\section{Results and discussion}

After the first blood patch procedure $87 \%$ of patients obtained complete symptom regression within 3 months.

In $13 \%$ of patients the first EPB was not effective and a second treatment has been performed reaching a global 
success rate of $90 \%$. So $10 \%$ of patients could not be cured by the blood patch procedure and subsequently developed chronic tensive headache refractory to drugs and characterized by the progressive disappearance of the postural changes typical of the SIH.

Only 2 out of 15 patients with convexity fluid collections (Fig. 1) requested surgical evacuation after the BP procedure.

Finally, it has to be remarked that $25 \%$ of patients had recurrence of the disease within 2-8 years after the BP procedure, which was then was successfully repeated.

Some topics from our experience in the reported series are remarked:

- All patients at hospital admission were highly invalidated and after the blood patch procedures $90 \%$ returned to normal life in few weeks.

- None of our patients needed open surgery to repair CSF leak.

- There is no need to search the radiological evidence and site of the CSF leak; in fact brain MRI and clinical data are enough to establish the diagnosis of SIH.

- The therapeutic mechanism of the epidural lumbar blood patch is related to the rise of the spinal epidural pressure and to the correction of the negative balance between CSF and epidural space. The success rate of EBP in patients who underwent diagnostic procedures aimed to discover the CSF leak (47\%) and the success rate in patients who underwent lumbar EBP following the clinical and brain MRI data was the same.

In conclusion, lumbar BP was effective independently of the severity of the disease and the site of the CSF leak, thus confirming that the negative epidural pressure plays a major role in the pathogenesis of SIH.

Epidural lumbar blood patch is the procedure of choice for SIH when conservative treatments failed. Anyway $10 \%$ of patients develop chronic headache syndromes in spite of repeated BP procedures.
Acknowledgments We gratefully acknowledgement Eliana Mea MD for the support.

Conflict of interest We certify that there is no actual or potential conflict of interest in relation to this article.

\section{References}

1. Berroir S, Loisel B, Ducros A, Boukobza M, Tzourio C, Valade D, Bousser MG (2004) Early epidural blood patch in spontaneous intracranial hypotension. Neurology 63(10):1950-1951

2. Chiapparini L, Ciceri E, Nappini S, Castellani MR, Mea E, Bussone G, Leone M, Savoiardo M (2004) Headache and intracranial hypotension: neuroradiological findings. Neurol Sci 25(Suppl 3):138-141

3. Dillo W, Hollenhorst J, Brassel F, von Hof-Strobach K, Heidenreich F, Johannes S (2002) Successful treatment of a spontaneous cervical cerebrospinal fluid leak with a CT guided epidural blood patch. J Neurol 249:224-225

4. Ferrante E, Arpino I, Citterio A, Wetzl R, Savino A (2010) Epidural blood patch in Trendelenburg position pre-medicated with acetazolamide to treat spontaneous intracranial hypotension. Eur J Neurol 17(5):715-719

5. Franzini A, Messina G, Nazzi V, Mea E, Leone M, Chiapparini L, Broggi G, Bussone G (2010) Spontaneous intracranial hypotension syndrome: a novel speculative physiopathological hypothesis and a novel patch method in a series of 28 consecutive patients. J Neurosurg 112:300-306

6. Franzini A, Zekaj E, Messina G, Mea E, Broggi G (2010) Intracranial spontaneous hypotension associated with CSF cervical leakage successfully treated by lumbar epidural blood patch. Acta Neurochir (Wien). 152(11):1997-1999

7. Mea E, Franzini A, D'Amico D, Leone M, Cecchini AP, Tullo V, Chiapparini L, Bussone G (2011) Treatment of alterations in CSF dynamics. Neurol Sci. 32(Suppl 1):117-120

8. Mokri B (2003) Headaches caused by decreased intracranial pressure: diagnosis and management. Curr Opin Neurol 16: 319-326

9. Rai A, Rosen C, Carpenter J, Miele V (2005) Epidural blood patch at $\mathrm{C} 2$ : diagnosis and treatment of spontaneous intracranial hypotension. AJNR Am J Neuroradiol 26(10):2663-2666

10. Schievink WI (2000) Spontaneous spinal cerebrospinal fluid leaks: a review. Neurosurg Focus 9(1) (review)

11. Sencakova D, Mokri B, McClelland RL (2001) The efficacy of epidural blood patch in spontaneous CSF leaks. Neurology 57:1921-1923 